\title{
ALLOPHENYLNORSTATINE-CONTAINING HIV-1 PROTEASE INHIBITORS: DESIGN, SYNTHESIS AND STRUCTURE-ACTIVITY RELATIONSHIPS FOR SELECTED $P_{2}$ LIGANDS
}

\author{
Hamdy M. Abdel-Rahman ${ }^{1}$, Nawal A. El-Koussi ${ }^{* 1}$, Gamal S. Alkaramany ${ }^{1}$, Adel F. Youssef ${ }^{1}$ \\ and Yoshiaki Kiso ${ }^{2}$ \\ ${ }^{* 1}$ Department of Pharmaceutical Medicinal Chemistry, Faculty of Pharmacy, Assiut \\ University, Assiut 71526, Egypt \\ ${ }^{2}$ Department of Medicinal Chemistry, Kyoto Pharmaceutical University, Kyoto, 607-8412 \\ Japan
}

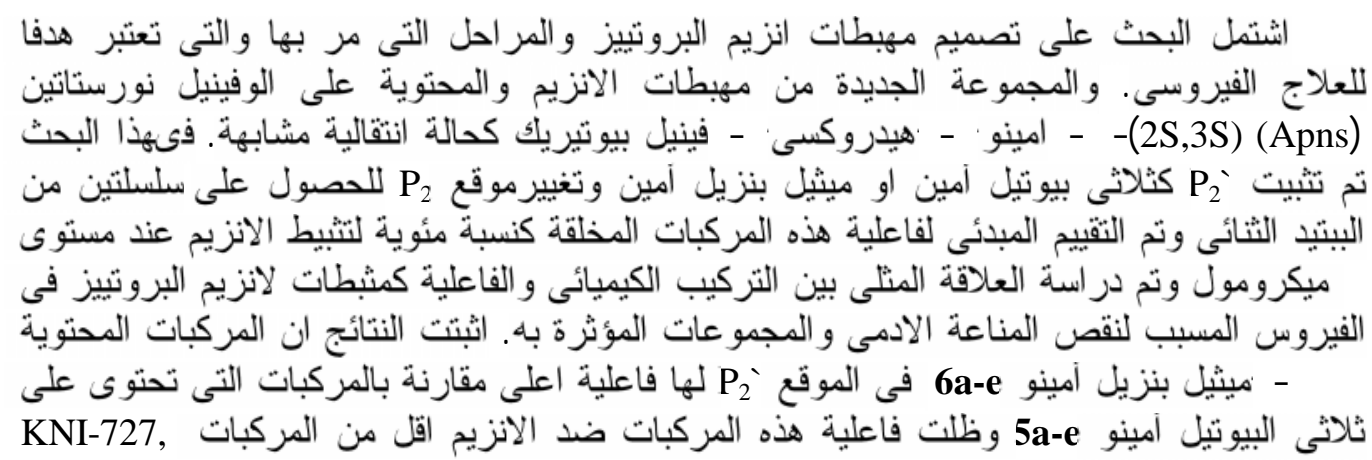

KNI-577

The design and development of potent HIV protease inhibitors remain an attractive target for antiviral therapy. A novel class of HIV protease inhibitors containing allophenylnorstatine [Apns; (2S,3S)-3-amino-2-hydroxy-4-phenylbutyric acid] as a transition state mimic have been reported. In this work we fixed $P_{2}{ }^{\prime}$ (as tert-butylamino or 2-methylbenzylamino) and changed $P_{2}$ moiety to provide two series of dipeptide analogs. Preliminary evaluation of the activity of the synthesized derivatives were determined as percentage of enzyme inhibition at $5 \mu M$ level. The results showed that the introduction of 2-methylbenzylamino moiety as $P_{2}$ 'ligand 6a-e considerably improved HIV inhibitory activity in comparison with the tert-butyl amino analogs 5a-e. It was found that compounds in both series retained activity still less than the lead compounds KNI-577 and KNI-727.

\section{INTRODUCTION}

The human immunodeficiency virus (HIV) encode an aspartic protease that processes polyprotein precursors into viral structural proteins and replicative enzymes. This processing is essential for assembly and maturation of fully infectious virions. Thus the design of inhibitors of HIV protease is an important therapeutic target in the treatment of AIDS. ${ }^{1}$ Incorporation of allophenylnorstatine in Phe-Pro peptidomimetic inhibitors of HIV protease has led to the development of a novel series of highly potent antiviral compounds, represented by the tripeptide compound KNI-
272 which possesses excellent characteristics of protease inhibition, antiviral activity, enzyme selectivity, and low toxicity. ${ }^{2}$

Trials to improve in vivo behavior of the tripeptide KNI-272, have led to the preparation of the truncated dipeptide HIV protease inhibitors with potent enzyme inhibitory activity like KNI-577 and KNI-727.,

In this work we are interested in relating the structure properties of two series of dipeptides with their activity as HIV protease inhibitors. Our strategy was based on variation of $\mathrm{P}_{2}$ moiety while fixing the substituent in $\mathrm{P}_{2}$ as tert-butylamino in one of the series and as 2methylbenzylamino in the second. 
<smiles>[R5]P[PH2]N[C@@H](Cc1ccccc1)[C@H](O)C(=O)N1CSC([R7])(P)[C@H]1C(=O)NC(C)(C)C</smiles>

KNI-272<smiles>CC(=O)COc1cccc2cnccc12</smiles><smiles>CSC[C@H](NC=P)C(C)=O</smiles><smiles>CP=Cc1cccc(O)c1C</smiles>

$\mathrm{KNI}-727$<smiles>CC(=O)COc1cc(P)ccc1C</smiles>

\section{EXPERIMENTAL}

\section{Chemistry}

Melting points were determined on a micro hot plate of Yanaco micro melting point apparatus and are uncorrected. The optical rotations were measured on Horiba model SEPA-300 digital polarimeter. TLC was performed on precoated Merck silica gel 60 $\mathrm{F}_{254}$ sheets. Column chromatography was carried out on Merck Silica gel 60 (particle size 0.063-0.200 mm).

Analytical RP-HPLC was performed by Hitachi L-7100 pump and L-7400 U.V. detector utilizing YMC Pack ODS-AM AM 302.

Preparative RP-HPLC was performed by Shimadzu LC-4A liquid chromatograph utilizing a YMC Pack ODS-AM type SH-3435AM column $(250$ x 20 mmI.D., S-5 $\mu \mathrm{m}$, 120A).

${ }^{1} \mathrm{H}$ NMR spectra were recorded on JEOL JNM-EX $270 \quad(270 \quad \mathrm{MHz}) \quad$ Spectrometer. Chemical shifts are given in $(\delta \mathrm{ppm})$ relative to tetramethylsilane (TMS) as an internal standard. FAB mass spectra (FAB-MS) and high resolution FAB-MS (HRFAB-MS) were recorded on JEOL JMS-SX102 AQQ/MSHYB10 mass spectrometer using glycerol, thioglycerol, or Magic Bullet as internal references. MALDI TOF Mass Spectra were measured at Voyager-DE ${ }^{\mathrm{TM}} \mathrm{RP}$ Biospectrometry ${ }^{\mathrm{TM}}$ Workstation (PerSeptive Biosystems). Commercially available chemicals were purchased from Nacalai tesque, Waku Chemicals or Tokyo Chemical Industries, Japan and were used without further purification.

The following compounds are $\mathrm{P}_{2}$ ligands which were not available commercially and were prepared according to standard methods described in details in reference. ${ }^{5}$

i) N-Benzyloxycarbonylanthranilic acid was prepared by reaction of anthranilic acid with benzylchloroformate in presence of $\mathrm{NaHCO}_{3}$ (Yield 68\%, m.p 139 ${ }^{\circ}$ ).

ii) $\mathrm{N}$-(Substituted)phthalmic acid was prepared by reaction of phthalic anhydride with the corresponding amines in chloroform.

$\mathrm{N}$-(2-pyridyl)phthalmic acid (Yield 84\%, m.p $\left.164-165^{\circ}\right)$;

N-(3-pyridyl)phthalmic acid (Yield 94\%, m.p $180-182^{\circ}$ );

$\mathrm{N}$-(4-pyridyl)phthalmic acid (Yield 87\%, m.p 250-252 ${ }^{\circ}$.

(2S,3S)3-(tert-Butyloxycarbonyl)amino-2hydroxy-4-phenylbutanoic acid; N-BocApns-OH,${ }^{6,7}$ Yield (8\%), m.p147-148 .

N-Boc-5,5-dimethylthiazolidine-3-carboxylic acid; (Boc-Dmt-OH) $2^{8}$ Yield (81\%), m.p $124-127^{\circ}$. 
N-tert-Butyl(tert-butyloxycarbonyl)-5,5dimethylthiazolidine-4-carboxamide; BocDmt-NH-tert-butyl 3. ${ }^{5}$

To a solution of the appropriate carboxylic acid $(20 \mathrm{mmol})$ in DMF-CHCl 3 (1:1 mixture) $(40 \mathrm{ml}), \mathrm{HOBt} . \mathrm{H}_{2} \mathrm{O}(3.67 \mathrm{~g}, 24 \mathrm{mmol})$ and DCC.HCl (4.95 g, $24 \mathrm{mmol})$ were added during stirring at $0^{\circ}$. The reaction mixture was stirred for $20 \mathrm{~min}$ at room temperature. tertbutylamine $(4.20 \mathrm{ml}, 40 \mathrm{mmol})$ was added, and stirring was continued for $5 \mathrm{~h}$ at room temperature then DCU was filtered off and the solvents were evaporated under reduced pressure. The residue was extracted with AcOEt and washed with citric acid, $\mathrm{NaHCO}_{3}$, and brine then dried over anhydrous $\mathrm{Na}_{2} \mathrm{SO}_{4}$, filtered, the filtrate evaporated under reduced pressure. The crude product, Table 1, was crystallized from $n$-hexane and dried under vacuum.

Table 1: Physical data of intermediate compounds 3-6.

\begin{tabular}{|c|c|c|c|c|}
\hline No. & $\mathrm{P}_{2}{ }^{\prime}$ & $\mathrm{m} \cdot \mathrm{p}^{\circ}$ & $\begin{array}{l}\text { Method } \\
\text { of } \\
\text { coupling }\end{array}$ & $\begin{array}{l}\mathrm{HPLC}^{\mathrm{a})} \\
\mathrm{Rt}(\mathrm{min})\end{array}$ \\
\hline 3 & & $110-111$ & A & 26.28 \\
\hline 4 & & $139-141$ & D & 23.36 \\
\hline 5 & & $85-88$ & B & 22.38 \\
\hline 6 & & $77-80$ & E & 24.40 \\
\hline
\end{tabular}

a) HPLC system $20-80 \% \mathrm{CH}_{3} \mathrm{CN}$ in $0.1 \%$ aqueous THF over $30 \mathrm{~min}$.

\section{N-2-Methylbenzyl(tert-butyloxycarbonyl)- 5,5-dimethylthiazolidine-4-carboxamide Boc-Dmt-NH-2-methylbenzyl 4}

Boc-Dmt-OH 2 (1.5 g, $5.74 \mathrm{mmol})$ was dissolved and stirred in AcOEt $(20 \mathrm{ml}), \mathrm{Et}_{3} \mathrm{~N}$ $(0.88 \mathrm{ml}, 6.33 \mathrm{mmol})$ and DPPCl $(1.31 \mathrm{ml}, 6.33$ mmol) were added at $0^{\circ}$. The mixture was stirred at room temperature for $1 \mathrm{~h}$, then 2methylbenzylamine $(6.33 \mathrm{mmol})$ and $\mathrm{Et}_{3} \mathrm{~N}$ $(0.88 \mathrm{ml}, 6.33 \mathrm{mmol})$ were added. Stirring was continued at room temperature for $6 \mathrm{~h}$. The mixture was washed twice with $10 \%$ citric acid, 5\% $\mathrm{NaHCO}_{3}$ and brine. Dried over anhydrous $\mathrm{Na}_{2} \mathrm{SO}_{4}$ and filtered. The filtrate was evaporated under reduced pressure, crystallized from n-hexane and dried, Table 1.

\section{N-tert-Butyl-3-(2S,3S)-3-(tert-butyloxy-} carbonyl)amino-2-hydroxy-4-phenyl butanoyl)-5,5-dimethylthiazolidine-4carboxamide; Boc-Apns-Dmt-NH-tertbutyl $5^{2,5}$

To a solution of the appropriate Bocpeptide $(20.8 \mathrm{mmol})$ in $4 \mathrm{~N} \mathrm{HCl} /$ Dioxane $(40$ $\mathrm{ml}$ ) anisol (4.5 ml, $41.67 \mathrm{mmol})$ was added at $0^{\circ}$. The reaction mixture was stirred for $1 \mathrm{~h}$ at room temperature. The solvent was removed in vacuo at room temperature, ether was added and the mixture was centrifuged. The formed precipitate was dissolved in DMF $(40 \mathrm{ml})$ and the respective carboxylic acid $(22.85 \mathrm{mmol})$, HOBt. $\mathrm{H}_{2} \mathrm{O}$ (3.5 g, $\left.22.85 \mathrm{mmol}\right)$, EDC.HCl (4.3 $\mathrm{g}, 22.43 \mathrm{mmol})$ and $\mathrm{Et}_{3} \mathrm{~N}(5.78 \mathrm{ml}, 41.6 \mathrm{mmol})$ were added at $0^{\circ}$. The reaction mixture was stirred overnight at room temperature, and the solvent was removed under reduced pressure. The residue was extracted with AcOEt and the organic layer washed with $10 \%$ citric acid, 5\% $\mathrm{NaHCO}_{3}$ and brine dried over anhydrous $\mathrm{Na}_{2} \mathrm{SO}_{4}$, filtered, evaporated and the residue crystallized from n-hexane, Table 1.

\section{N-2-Methylbenzyl-3-(2S,3S)-3-(tert-butyl- oxycarbonyl)amino-2-hydroxy-4-phenyl- butanoyl)-5,5-dimethylthiazolidine-4- carboxamide; Boc-Apns-Dmt-NH-2-methyl- benzyl $6^{2,5}$}

To Boc-Dmt-NH-2-methylbenzyl. 4 (0.5 $\mathrm{mmol})$, in $4 \mathrm{~N} \mathrm{HCl} /$ dioxane solution $(2 \mathrm{ml})$, anisol $(108 \mu \mathrm{l}, 1 \mathrm{mmol})$ was added at $0^{\circ}$. This solution was stirred for $2 \mathrm{~h}$ at room temperature. The solvent was evaporated in vacuo, ether was added, the mixture was centrifuged and the residue was dissolved in DMF (5 ml). Boc-Apns-OH 3 (134 mg, 0.45 mmol), HOBt. $\mathrm{H}_{2} \mathrm{O}$ (76.6 mg, $\left.0.5 \mathrm{mmol}\right)$, BOP (211 mg, $0.5 \mathrm{mmol})$ and $\mathrm{Et}_{3} \mathrm{~N}(139 \mathrm{ml}, 1 \mathrm{mmol})$ were added at $0^{\circ}$. The mixture was stirred overnight at room temperature The solvent was then removed under vacuum and the residue was extracted with AcOEt. The organic layer was washed with $10 \%$ citric acid, $5 \% \mathrm{NaHCO}_{3}$ and brine. Dried over anhydrous $\mathrm{Na}_{2} \mathrm{SO}_{4}$ filtered and evaporated under reduced pressure. 
The residue was purified by column chromatography and the appropriate fractions were pooled and evaporated to yield the coupled peptide (Boc-Apns-Dmt-NH-2methylbenzyl) which was dried in desiccator, Table 1.

\section{$\mathbf{P}_{2}$-Apns-Dmt-NH-tert-Bu 5a-e}

To a solution of appropriate N-Bocpeptide 5 (20.8 $\mathrm{mmol})$ in $4 \mathrm{~N} \mathrm{HCl} /$ dioxane $(40$ $\mathrm{ml})$, anisole (4.5 ml, $41.67 \mathrm{mmol})$, was added at $0^{\circ}$. The reaction mixture was stirred for $1 \mathrm{~h}$ at room temperature. The solvent was removed in vacuo at room temperature, ether was added, and the mixture was centrifuged. The formed precipitate was dissolved in DMF $(40 \mathrm{ml})$, then the carboxylic acid $(22.85 \mathrm{mmol}), \mathrm{HOBt} . \mathrm{H}_{2} \mathrm{O}$ (3.5 g, $22.85 \mathrm{mmol})$, EDC.HCl (4.3 g, 22.43 $\mathrm{mmol})$ and $\mathrm{Et}_{3} \mathrm{~N}(5.78 \mathrm{ml}, 41.6 \mathrm{mmol})$ were added at $0^{\circ}$. The reaction mixture was stirred over night at room temperature, and the solvent was removed under reduced pressure. The residue was extracted AcOEt and the organic layer washed with $10 \%$ citric acid, 5\% $\mathrm{NaHCO}_{3}$ and brine, dried over anhydrous $\mathrm{Na}_{2} \mathrm{SO}_{4}$, filtered, evaporated and the residue crystallized from n-hexane. Physical data are listed in Table 2.

\section{Procedure of 5c-e}

To a solution of the appropriate BocApns-Dmt- ${ }_{2}{ }^{\prime}(0.1 \mathrm{mmol})$ in $4 \mathrm{~N} \mathrm{HCl} /$ Dioxane $(1 \mathrm{ml})$, anisol $(22 \mu \mathrm{l}, 0.2 \mathrm{mmol})$ was added at $0^{\circ}$. This mixture was stirred for $1 \mathrm{~h}$ at room temperature. The solvent was removed in vacuo at temperature, ether was added and the mixture was centrifuged. The formed precipitate was dissolved in THF $(5 \mathrm{ml})$ and the respective carboxylic acid $(0.11 \mathrm{mmol})$, HOBt. $\mathrm{H}_{2} \mathrm{O}$ (16.8 mg, $0.11 \mathrm{mmol}$ ), EDC.HCl (33 mg, $0.172 \mathrm{mmol})$ and $\mathrm{Et}_{3} \mathrm{~N}(34 \mu \mathrm{l}, 0.2$ mmol) were added at $0^{\circ}$. The reaction mixture was stirred for $24 \mathrm{~h}$ at room temperature. The reaction was quenched with brine and the reaction mixture was extracted with AcOEt. The organic layer was washed with brine and dried over anhydrous $\mathrm{Na}_{2} \mathrm{SO}_{4}$, filtered, evaporated and crystallized from $n$-hexane. The compounds obtained after crystallization were checked by analytical HPLC, from the resulting data preparative HPLC was established and was carried out. The purity was checked again by analytical HPLC. The fractions were mixed and lyophilized to afford the analytically pure final compounds so yields of the products were determined by reversed phase HPLC (RPHPLC). Physical data are listed in Table 2.

\section{2-(7-Methoxy)benzofuranoyl-Apns-Dmt- NH-tert-Bu 5a}

$[\alpha]_{\mathrm{D}}{ }^{25}-68.00\left(\mathrm{c}=0.25, \mathrm{CH}_{3} \mathrm{OH}\right),{ }^{1} \mathrm{H}$ NMR $\left(\right.$ DMSO-d $\left._{6}\right) \delta: 1.25(\mathrm{~s}, 9 \mathrm{H}), 1.40(\mathrm{~s}, 3 \mathrm{H}), 1.49$ (s, 3H), 2.77-2.98 (m, 2H), 3.95 (s, 3H), 4.44 (bs, 1H), 4.50-4.54 (m, 2H), 4.94-4.97 (d, $J=$ $8.91 \mathrm{~Hz}, 1 \mathrm{H}), 5.03-5.06(\mathrm{~d}, J=8.58 \mathrm{~Hz}, 1 \mathrm{H})$, 7.03-7.31 (m, 6H), 7.38-7.41 (d, $J=7.91 \mathrm{~Hz}$, $2 \mathrm{H}), 7.52(\mathrm{~s}, 1 \mathrm{H}), 7.62(\mathrm{~s}, 1 \mathrm{H}), 8.62-8.65(\mathrm{~d}, J=$ $8.25 \mathrm{~Hz}, 1 \mathrm{H})$, HRFAB-MS: $\mathrm{m} / \mathrm{z} 568.2493$ for $[\mathrm{M}+\mathrm{H}]^{+}$(calcd. 568.2481 for $\mathrm{C}_{30} \mathrm{H}_{38} \mathrm{~N}_{3} \mathrm{O}_{6} \mathrm{~S}$ ).

\section{N-(Benzyloxycarbonyl)-2-aminobenzoyl- Apns-Dmt-NH-tert-Bu 5b}

$[\alpha]_{\mathrm{D}}{ }^{27}-3.65\left(\mathrm{c}=0.26, \mathrm{CH}_{3} \mathrm{OH}\right),{ }^{1} \mathrm{H} \mathrm{NMR}$ (DMSO-d $\left.\mathrm{d}_{6}\right) \delta: 1.22(\mathrm{~s}, 9 \mathrm{H}), 1.37(\mathrm{~s}, 3 \mathrm{H}), 1.44$ (s, 3H), 2.75-2.84 (m, 2H), 4.41-4.54 (m, 3H), 4.94-4.98 (d, $J=8.58 \mathrm{~Hz}, 1 \mathrm{H}), 5.04-5.07$ (d, $J=$ $8.91 \mathrm{~Hz}, 1 \mathrm{H}), 5.12(\mathrm{~s}, 2 \mathrm{H}), 7.04-7.09$ (t, $J=$ $7.59 \mathrm{~Hz}, 2 \mathrm{H}), 7.15-7.20(\mathrm{t}, J=7.59 \mathrm{~Hz}, 2 \mathrm{H})$, 7.33-7.48 (m, 8H), $7.58(\mathrm{~s}, 1 \mathrm{H}), 7.67-7.70(\mathrm{~d}$, $J=7.91 \mathrm{~Hz}, 1 \mathrm{H}), 8.10-8.13(\mathrm{~d}, J=8.58 \mathrm{~Hz}, 1 \mathrm{H})$, 8.66-8.69 (d, $J=8.58 \mathrm{~Hz}, 1 \mathrm{H}), 10.53(\mathrm{~s}, 1 \mathrm{H})$, HRFAB-MS: $m / z, 647.2902$ for $[\mathrm{M}+\mathrm{H}]^{+}$(calcd. 647.2903 for $\mathrm{C}_{35} \mathrm{H}_{43} \mathrm{~N}_{4} \mathrm{O}_{6} \mathrm{~S}$ ).

\section{N-(2-Pyridylmethyl)phthalamoyl-Apns- Dmt-NH-tert-Bu 5c}

$[\alpha]_{\mathrm{D}}{ }^{24.5}+13.34\left(\mathrm{c}=0.067, \mathrm{CH}_{3} \mathrm{OH}\right),{ }^{1} \mathrm{H}$ NMR (DMSO-d $\left.)_{6}\right) \delta: 1.26(\mathrm{~s}, 9 \mathrm{H}), 1.40(\mathrm{~s}, 3 \mathrm{H})$, 1.47 (s, 3H), 2.60-2.80 (m, 2H), 4.26 (bs, $1 \mathrm{H})$, 4.37-4.44 (m, 1H), $4.52(\mathrm{~s}, 1 \mathrm{H}), 4.60-4.75(\mathrm{~m}$, $3 \mathrm{H}), 5.08-5.11(\mathrm{~d}, J=9.23 \mathrm{~Hz}, 1 \mathrm{H}), 7.14-7.29$ (m, 4H), 7.40-7.52 (m, 6H), 7.66-7.68 (m, 2H), 7.85-7.95 (t, $J=6.6 \mathrm{~Hz}, 1 \mathrm{H}), 8.55-8.57(\mathrm{~d}, J=$ $5.28 \mathrm{~Hz}, 1 \mathrm{H}), 8.68-8.72(\mathrm{~d}, J=8.25 \mathrm{~Hz}, 1 \mathrm{H})$, 8.85-8.90 (t, $J=5.8 \mathrm{~Hz}, 1 \mathrm{H})$, HRFAB-MS: $\mathrm{m} / \mathrm{z}$ 632.2901 for $[\mathrm{M}+\mathrm{H}]^{+}$(calcd. 632.2907 for $\mathrm{C}_{34} \mathrm{H}_{42} \mathrm{~N}_{5} \mathrm{O}_{5} \mathrm{~S}$ ).

\section{N-(3-Pyridylmethyl)phthalamoyl-Apns- Dmt-NH-tert-Bu 5d}

$[\alpha]_{\mathrm{D}}{ }^{25}+30.00\left(\mathrm{c}=0.1, \mathrm{CH}_{3} \mathrm{OH}\right),{ }^{1} \mathrm{H}$ NMR $\left(\right.$ DMSO-d $\left._{6}\right) \delta: 1.26(\mathrm{~s}, 9 \mathrm{H}), 1.40(\mathrm{~s}, 3 \mathrm{H}), 1.47$ (s, 3H), 2.74-2.88 (m, 2H), 4.26 (bs, 1H), 4.36$4.44(\mathrm{~m}, 1 \mathrm{H}), 4.52-4.68(\mathrm{~m}, 3 \mathrm{H}), 4.71-4.75(\mathrm{~d}$, $J=8.58 \mathrm{~Hz}, 1 \mathrm{H}), 5.04-5.07(\mathrm{~d}, J=8.25 \mathrm{~Hz}, 1 \mathrm{H})$, 
Table 2: Physical data of dipeptide-based HIV protease inhibitors $\left(\mathrm{P}_{2}^{\prime}=\right.$ tert-butylamino $)$.

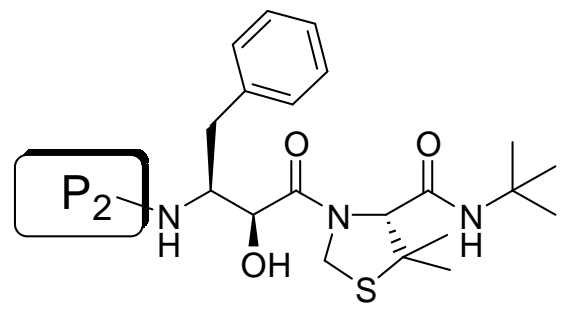

\begin{tabular}{|c|c|c|c|c|c|c|c|}
\hline \multirow{2}{*}{ No. } & \multirow{2}{*}{$\mathrm{P}_{2}$} & \multirow{2}{*}{$\mathrm{m} \cdot \mathrm{p}^{\circ}$} & \multirow{2}{*}{$\begin{array}{l}\text { Yield } \\
(\%)\end{array}$} & \multirow{2}{*}{$\begin{array}{l}\text { Method } \\
\text { of } \\
\text { coupling }\end{array}$} & \multirow{2}{*}{$\begin{array}{l}\operatorname{HPLC}^{a)} \\
\operatorname{Rt}(\min )\end{array}$} & \multicolumn{2}{|c|}{ TLC } \\
\hline & & & & & & $\mathrm{R} f_{1}^{b)}$ & $\mathrm{R} f_{2}^{\left({ }^{c}\right)}$ \\
\hline $5 \mathbf{a}$ & & $113-116$ & 81 & $\mathrm{C}$ & 23.62 & 0.86 & 0.94 \\
\hline $5 \mathbf{b}$ & & $99-101$ & 73 & B & 28.74 & 0.65 & 0.87 \\
\hline $5 c$ & & $127-130$ & 82 & $\mathrm{C}$ & 15.74 & 0.56 & 0.80 \\
\hline $5 d$ & & $141-144$ & 95 & $\mathrm{C}$ & 16.36 & 0.50 & 0.78 \\
\hline $5 \mathbf{e}$ & & $143-146$ & 79 & $\mathrm{C}$ & 16.18 & 0.47 & 0.69 \\
\hline
\end{tabular}

a) $20-80 \% \mathrm{CH}_{3} \mathrm{CN}$ in $0.1 \%$ aqueous TFA over 30 min.,

b) $\mathrm{CHCl}_{3}: \mathrm{CH}_{3} \mathrm{OH}(10: 1)$, c) $\mathrm{CHCl}_{3}: \mathrm{CH}_{3} \mathrm{OH}: \mathrm{H}_{2} \mathrm{O}(8: 3: 1)$

7.15-7.31 (m, 4H), 7.40-7.43 (d, $J=7.26 \mathrm{~Hz}$, $2 \mathrm{H}), 7.49(\mathrm{~m}, 3 \mathrm{H}), 7.68(\mathrm{~m}, 2 \mathrm{H}), 8.21-8.24(\mathrm{~d}$, $J=7.59 \mathrm{~Hz}, 1 \mathrm{H}), 8.66-8.69(\mathrm{~m}, 2 \mathrm{H}), 8.76(\mathrm{~s}$, $1 \mathrm{H}), 8.88$ (t, $J=5.8 \mathrm{~Hz}, 1 \mathrm{H})$, HRFAB-MS: $\mathrm{m} / \mathrm{z}$ 632.2891 for $[\mathrm{M}+\mathrm{H}]^{+}$(calcd. 632.2907 for $\mathrm{C}_{34} \mathrm{H}_{42} \mathrm{~N}_{5} \mathrm{O}_{5} \mathrm{~S}$ ).

\section{N-(4-Pyridylmethyl)phthalamoyl-Apns- \\ Dmt-NH-tert-Bu 5e}

$[\alpha]_{\mathrm{D}}{ }^{23}+14.00\left(\mathrm{c}=0.15, \mathrm{CH}_{3} \mathrm{OH}\right),{ }^{1} \mathrm{H}$ NMR $\left(\right.$ DMSO-d $\left._{6}\right) \delta 1.26(\mathrm{~s}, 9 \mathrm{H}), 1.41(\mathrm{~s}, 3 \mathrm{H}), 1.47$ (s, 3H), 2.75-2.87 (m, 2H), 4.27 (bs, 1H), 4.46$4.52(\mathrm{~m}, 2 \mathrm{H}), 4.60(\mathrm{~s}, 1 \mathrm{H}), 4.66-4.73(\mathrm{~m}, 2 \mathrm{H})$, 5.08-5.11 (d, $J=8.9 \mathrm{~Hz}, 1 \mathrm{H}), 7.14-7.33(\mathrm{~m}$, 4H), 7.41-7.44 (d, J= 7.92 Hz, 2H), 7.48-7.51 $(\mathrm{m}, 3 \mathrm{H}), 7.68(\mathrm{~s}, 1 \mathrm{H}), 7.81-7.83(\mathrm{~d}, J=5.28 \mathrm{~Hz}$, $2 \mathrm{H}), 8.69-8.73(\mathrm{~m}, 3 \mathrm{H}), 8.90-8.94$ (t, $J=5.61$
$\mathrm{Hz}, \quad 1 \mathrm{H})$, HRFAB-MS: $\mathrm{m} / \mathrm{z} \quad 632.2916$ for $[\mathrm{M}+\mathrm{H}]^{+}$(calcd. 632.2907 for $\mathrm{C}_{34} \mathrm{H}_{42} \mathrm{~N}_{5} \mathrm{O}_{5} \mathrm{~S}$ ).

\section{Synthesis of $\mathbf{P}_{2}$-Apns-Dmt-NH-(2-Me)Bz 6a-e}

The titled compounds were prepared, starting from N-Boc-Apns-Dmt-NH-(2-Me) Bz 6 by removal of Boc then coupling with the corresponding $\mathrm{P}_{2}$ carboxylic acid, as described under the preparation of $\mathbf{5}$. Physical data are listed in Table 3.

\section{2-(7-Methoxy)benzofuranoyl-Apns-Dmt- NH-(2-Me)Bz 6a}

$[\alpha]_{\mathrm{D}}{ }^{24.5}-58.60 \quad\left(\mathrm{c}=0.43, \mathrm{CH}_{3} \mathrm{OH}\right),{ }^{1} \mathrm{H}$ NMR (DMSO-d $\left.{ }_{6}\right) \delta: 1.36(\mathrm{~s}, 3 \mathrm{H}), 1.52(\mathrm{~s}, 3 \mathrm{H})$, 2.20 (s, 3H), 2.80-3.00 (m, 2H), 3.92 (s, 3H), 
Table 3: Physical data of dipeptide-based HIV protease inhibitors $\mathrm{P}_{2}{ }^{\prime}=2-(\mathrm{Me})$ benzylamino.<smiles>[2H]C([2H])N[C@@H](Cc1ccccc1)[C@H](O)C(=O)N1CSC(C)(C)[C@H]1C(=O)NCc1ccccc1C</smiles>

\begin{tabular}{|c|c|c|c|c|c|c|c|}
\hline \multirow{2}{*}{ No. } & \multirow{2}{*}{$\mathrm{P}_{2}$} & \multirow{2}{*}{$\mathrm{m} \cdot \mathrm{p}^{\circ}$} & \multirow{2}{*}{$\begin{array}{c}\text { Yield } \\
(\%)\end{array}$} & \multirow{2}{*}{$\begin{array}{l}\text { Method of } \\
\text { coupling }\end{array}$} & \multirow{2}{*}{$\begin{array}{l}\left.\mathrm{HPLC}^{a}\right) \\
\mathrm{Rt}(\min )\end{array}$} & \multicolumn{2}{|c|}{ TLC } \\
\hline & & & & & & $\mathrm{R} f_{1}^{b)}$ & $\mathrm{R} f_{2}^{(c)}$ \\
\hline $\mathbf{6 a}$ & & $110-112$ & 73 & $\mathrm{C}$ & 24.45 & 0.87 & 0.94 \\
\hline $\mathbf{6 b}$ & & $96-99$ & 65 & $\mathrm{C}$ & 28.29 & 0.90 & 0.94 \\
\hline $6 c$ & & $117-118$ & 64.5 & $\mathrm{C}$ & 16.50 & 0.85 & 0.93 \\
\hline 6d & & $125-127$ & 82.8 & $\mathrm{C}$ & 15.93 & 0.53 & 0.75 \\
\hline $5 e$ & & $130-132$ & 73.6 & $\mathrm{C}$ & 15.78 & 0.51 & 0.66 \\
\hline
\end{tabular}

a), b) and c) are the same as given under Table 2 .

4.04-4.15 (m, 1H), 4.29-4.41 (m, 1H), 4.49$4.53(\mathrm{~m}, 3 \mathrm{H}), 4.96-5.03(\mathrm{~m}, 2 \mathrm{H}), 7.02-7.34(\mathrm{~m}$, $12 \mathrm{H}), 7.49(\mathrm{~s}, 1 \mathrm{H}), 8.28(\mathrm{~s}, 1 \mathrm{H}), 8.60(\mathrm{~m}, 1 \mathrm{H})$, HRFAB-MS: $m / z 616.2476$ for $[\mathrm{M}+\mathrm{H}]^{+}$(calcd. 616.2481 for $\mathrm{C}_{34} \mathrm{H}_{38} \mathrm{~N}_{3} \mathrm{O}_{6} \mathrm{~S}$ ).

\section{$\mathrm{N}$-(Benzyloxycarbonyl)-2-aminobenzoyl- Apns-Dmt-NH-(2-Me)Bz 6b} $[\alpha]_{\mathrm{D}}{ }^{23.5}+4.00 \quad\left(\mathrm{c}=0.125, \mathrm{CH}_{3} \mathrm{OH}\right),{ }^{1} \mathrm{H}$ NMR (DMSO-d $\left.\mathrm{d}_{6}\right) \delta: 1.32(\mathrm{~s}, 3 \mathrm{H}), 1.46(\mathrm{~s}, 3 \mathrm{H})$, $2.24(\mathrm{~s}, 3 \mathrm{H}), 2.63-2.89(\mathrm{~m}, 2 \mathrm{H}), 4.05-4.20(\mathrm{~m}$, $1 \mathrm{H})$, 4.34-4.50 (m, 4H), 4.98-5.12 (m, 4H), 7.10-7.20 (m, 6H), 7.29-7.46 (m, 9H), 7.66$7.69(\mathrm{~d}, J=7.91 \mathrm{~Hz}, 1 \mathrm{H}), 8.12-8.15(\mathrm{~d}, J=8.57$ $\mathrm{Hz}, 1 \mathrm{H}), 8.32(\mathrm{t}, J=5.61 \mathrm{~Hz}, 1 \mathrm{H}), 8.64-8.67(\mathrm{~d}$, $J=8.57 \mathrm{~Hz}, 1 \mathrm{H}), 10.55(\mathrm{~s}, 1 \mathrm{H})$, HRFAB-MS: $\mathrm{m} / \mathrm{z} 695.2907$ for $[\mathrm{M}+\mathrm{H}]^{+}$(calcd. 695.2903 for $\mathrm{C}_{39} \mathrm{H}_{43} \mathrm{~N}_{4} \mathrm{O}_{6} \mathrm{~S}$ ).

\section{N-(2-Pyridylmethyl)phthalamoyl-Apns-}

\section{Dmt-NH-(2-Me)Bz 6c}

$[\alpha]_{\mathrm{D}}^{25.1}+15.49 \quad\left(\mathrm{c}=0.25, \mathrm{CH}_{3} \mathrm{OH}\right),{ }^{1} \mathrm{H}$ NMR (DMSO-d $\left.\mathrm{d}_{6}\right) \delta: 1.35(\mathrm{~s}, 3 \mathrm{H}), 1.49(\mathrm{~s}, 3 \mathrm{H})$, $2.24(\mathrm{~s}, 3 \mathrm{H}), 2.75-2.80(\mathrm{~m}, 2 \mathrm{H}), 4.04(\mathrm{~m}, 1 \mathrm{H})$, 4.20-4.49 (m, 4H), 4.55-4.60 (m, 2H), 4.79$4.82(\mathrm{~d}, J=8.57 \mathrm{~Hz}, 1 \mathrm{H}), 5.04-5.06(\mathrm{~d}, \mathrm{~J}=8.57$ $\mathrm{Hz}, 1 \mathrm{H}), 7.08-7.26(\mathrm{~m}, 8 \mathrm{H}), 7.34-7.50(\mathrm{~m}, 6 \mathrm{H})$, $7.67(\mathrm{~d}, J=9.7 \mathrm{~Hz}, 1 \mathrm{H}), 7.88(\mathrm{t}, J=9.1 \mathrm{~Hz}$, $1 \mathrm{H}), 8.39(\mathrm{t}, J=5.6 \mathrm{~Hz}, 1 \mathrm{H}), 8.57-8.61(\mathrm{~m}$, 2H), $8.87(\mathrm{~m}, 1 \mathrm{H})$, HRFAB-MS: $\mathrm{m} / \mathrm{z} 680.2919$ for $[\mathrm{M}+\mathrm{H}]^{+}$(calcd. 680.2907 for $\mathrm{C}_{38} \mathrm{H}_{42} \mathrm{~N}_{5} \mathrm{O}_{5} \mathrm{~S}$ ).

\section{N-(3-Pyridylmethyl)phthalamoyl-Apns- \\ Dmt-NH-(2-Me)Bz 6d}

$[\alpha]_{\mathrm{D}}{ }^{25}+15.60\left(\mathrm{c}=0.25, \mathrm{CH}_{3} \mathrm{OH}\right),{ }^{1} \mathrm{H}$ NMR $\left(\right.$ DMSO-d $\left._{6}\right) \delta: 1.34(\mathrm{~s}, 3 \mathrm{H}), 1.48(\mathrm{~s}, 3 \mathrm{H}), 2.22$ (s, 3H), 2.75-2.93 (m, 2H), 3.97-4.01 (m, 1H), 
4.27 (bs, 1H), 4.35-4.42 (dd, $J=6.27,5.61 \mathrm{~Hz}$, $1 \mathrm{H}), 4.46-4.48(\mathrm{~d}, J=5.94 \mathrm{~Hz}, 2 \mathrm{H}), 4.57-4.65$ $(\mathrm{m}, 2 \mathrm{H}) 4.78-4.81(\mathrm{~d}, \mathrm{~J}=9.23 \mathrm{~Hz}, 1 \mathrm{H}), 4.99$ $5.02(\mathrm{~d}, J=9.24 \mathrm{~Hz}, 1 \mathrm{H}), 7.08-7.17(\mathrm{~m}, 4 \mathrm{H})$, 7.21-7.26 (m, 4H), 7.34-7.37 (d, J= 7.59 Hz, $2 \mathrm{H})$, 7.45-7.55 (m, 3H), 7.75-7.80 (m, $1 \mathrm{H})$, $8.28-8.31(\mathrm{~d}, \mathrm{~J}=7.58 \mathrm{~Hz}, 1 \mathrm{H}), 8.37$ (t, $J=5.61$ $\mathrm{Hz}, 1 \mathrm{H}), 8.55-8.58$ (d, $J=8.25 \mathrm{~Hz}, 1 \mathrm{H}), 8.69-$ $8.70(\mathrm{~d}, J=4.92 \mathrm{~Hz}, 1 \mathrm{H}), 8.79-8.83(\mathrm{~m}, 2 \mathrm{H})$, HRFAB-MS: $m / z 680.2919$ for $[\mathrm{M}+\mathrm{H}]^{+}$(calcd. 680.2907 for $\mathrm{C}_{38} \mathrm{H}_{42} \mathrm{~N}_{5} \mathrm{O}_{5} \mathrm{~S}$ ).

\section{N-(4-Pyridylmethyl)phthalamoyl-Apns- \\ Dmt-NH-(2-Me)Bz 6e \\ $[\alpha]_{\mathrm{D}}{ }^{26.5}+5.45\left(\mathrm{c}=0.11, \mathrm{CH}_{3} \mathrm{OH}\right),{ }^{1} \mathrm{H}$ NMR}

$\left(\right.$ DMSO-d $\left._{6}\right)$ 8: 1.35 (s, 3H), $1.48(\mathrm{~s}, 3 \mathrm{H}), 2.23$ (s, 3H), 2.75-2.94 (m, 2H), 3.97-4.04 (dd, $J=$ $3.96,4.61 \mathrm{~Hz}, 1 \mathrm{H}), 4.29$ (bs, 1H), 4.37-4.53 (m, $3 \mathrm{H}), 4.58-4.59(\mathrm{~d}, J=3.3 \mathrm{~Hz}, 1 \mathrm{H}), 4.63-4.75$ $(\mathrm{m}, 1 \mathrm{H}) 4.76-4.79(\mathrm{~d}, \mathrm{~J}=9.56 \mathrm{~Hz}, 1 \mathrm{H}), 5.01-$ $5.04(\mathrm{~d}, J=9.24 \mathrm{~Hz}, 1 \mathrm{H}), 7.11-7.36(\mathrm{~m}, 10 \mathrm{H})$, 7.48-7.51 (m, 3H), 7.86-7.89 (d, $J=5.94 \mathrm{~Hz}$, $2 \mathrm{H}), 8.37-8.39$ (t, $J=5.61 \mathrm{~Hz}, 1 \mathrm{H}), 8.60-8.63$ $(\mathrm{d}, J=7.92 \mathrm{~Hz}, 1 \mathrm{H}), 8.73-8.75(\mathrm{~d}, J=5.93 \mathrm{~Hz}$, $2 \mathrm{H}), 8.86-8.90(\mathrm{t}, J=5.61 \mathrm{~Hz}, 1 \mathrm{H})$, HRFABMS: $m / z \quad 680.2919$ for $[\mathrm{M}+\mathrm{H}]^{+}$(calcd. 680.2907 for $\mathrm{C}_{38} \mathrm{H}_{42} \mathrm{~N}_{5} \mathrm{O}_{5} \mathrm{~S}$ ).

\section{HIV protease inhibition assay}

Percentage of HIV protease inhibition was determined by an HPLC method using S10 peptide (H-Lys-Ala-Arg-Val-Tyr*Phe (p- $\left.\mathrm{NO}_{2}\right)$ Glu-Ala-Nle- $\mathrm{NH}_{2}$ ) as the enzyme substrate. The activities were tested at $5 \mu \mathrm{M}$ levels of the inhibitor. The assay protocol was that described by Mimoto et al. ${ }^{9}$

\section{RESULTS AND DISCUSSION}

\section{Chemistry}

Starting from L-penicillamine 1, N-Boc4,4-dimethylthiazolidine-3-carboxylic acid (Boc-Dmt-OH) 2 was obtained. ${ }^{8}$ After amidation of 2 with $\mathrm{P}_{2}-\mathrm{NH}_{2}$ residue, the yielded products $\mathbf{3 , 4}$ were converted to Apns intermediates $\mathbf{5 , 6 ^ { 2 , 5 }}$ as illustrated by Scheme 1 . The compounds 5,6 were used as scaffold for synthesis of the targeted dipeptides 5a-e and 6a-e. The same considerations as in classical peptide synthesis were adopted, but taken into account the suitable coupling agent and milieu of the reaction as described by the procedures A (DCC.HCl/ HOBt) ${ }^{10,11}$ B (EDC.HCl/HOBt in DMF), ${ }^{10,11} \mathrm{C}$ (EDC.HCl/HOBt in THF), ${ }^{10,11}$ $\mathrm{D}(\mathrm{DPPCl}){ }^{12} \mathrm{E}$ (BOP/HOBt in DMF). ${ }^{10,13}$ Reaction conditions were optimized in order to obtain good yields of the target compounds having satisfactory $\mathrm{m} / \mathrm{z}$ values compared with the calculated ones. Yields of the intermediates 3-6 and the target dipeptides 5a-e and 6a-e were determined after separation using gradiant preparative reversed-phase HPLC (RP-HPLC). Rt values, of the dipeptides 5a-e and 6a-e were shown in Tables 2 and 3 revealed wide variation in polarity that would presumably result in variation of water solubility which could be advantageous. Homogenity of the target dipeptides was monitored by TLC using two eluent system with significant different polarities.

\section{HIV protease inhibitory activity}

As a primary screen, dipeptide analogs were evaluated for inhibitory activity against HIV protease according to reported technique. ${ }^{9}$

The chosen $\mathrm{P}_{2}$ moieties in compounds (5be and $\mathbf{6 b}-\mathbf{e}$ ) were considered as rational mimics of the analog 3-hydroxy-2-methylbenzoyl group in KNI-577. N-acyl-2-aminobenzoyl 5b, $\mathrm{N}$-alkylphthalamoyl groups 5c-e seemed to be good candidates as $\mathrm{P}_{2}$ substitutes. On one hand they are readily synthesized, and non peptide in nature. On the other hand they provide the advantage of being simultaneously capable for hydrophobic and hydrogen bonding interactions with the complimentary sites of the enzyme.

The pyridyl moiety was incorporated at $\mathrm{P}_{2}$ site in the compounds $\mathbf{5 c - e}, \mathbf{6 c}-\mathbf{e}$ in order to improve the polarity. This was verified by the observed low Rt values (15.7-16.5) matched with the other derivatives which are devoid of the polar pyridine nucleus, while $\mathbf{5 a}, \mathbf{b}$ and $\mathbf{6 a}, \mathbf{b}$ showed Rt values in the range (23.6-28.74). The most active members in series $\mathbf{5}$ was $\mathbf{5 b}$ laking the pyridine moieties and showing the highest Rt values. The same pattern was detected in series $\mathbf{6}$ where $\mathbf{6 b}$ was the most active one, Table 4. On examining the results we can say that the site $\mathrm{P}_{2}$ can better tolerate lipophilic moieties, and enhancement of polarity might definitely reduce the protease inhibitory activity. Another searched point was the effect of changing $\mathrm{P}_{2}{ }^{\prime}$ on protease inhibition activity. Potential of HIV protease inhibition activity was significantly improved 
<smiles>CC(C)(S)[C@H](N)C(=O)O</smiles>

1

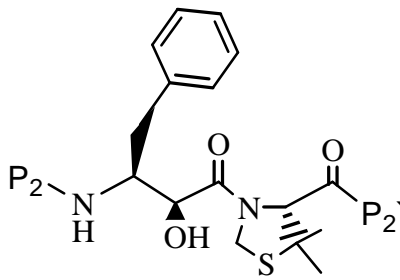

5a-e,6a-e vi, ix, viii

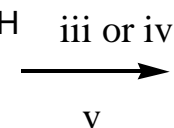

2<smiles>CC(C)(C)OC(=O)N1CSCC1C(=O)O</smiles>

3,4

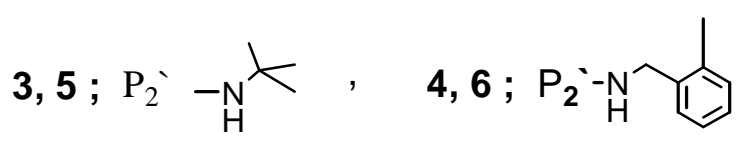

i: $37 \% \mathrm{HCHO}$; ii: $\left(\mathrm{Boc}_{2} \mathrm{O}_{2}, \mathrm{Et}_{3} \mathrm{~N}\right.$; iii: $\mathrm{DCC} / \mathrm{HOBt}$.in (DMF: $\mathrm{CHCl}_{3}$ )

iv: $\mathrm{DPPCl} / \mathrm{Et}_{3} \mathrm{~N} / \mathrm{AcOEt}$; v: $\mathrm{P}_{2}-\mathrm{NH}_{2}$; vi: $4 \mathrm{~N} \mathrm{HCl} /$ dioxane ; vii; $\mathrm{N}-\mathrm{Boc}-\mathrm{Apns}-\mathrm{OH}$

viii: $\mathrm{EDC}, \mathrm{HOBT} / \mathrm{DMF}$; ix: $\mathrm{P}_{2}--\mathrm{COOH}$

Scheme 1

Table 4: Inhibition potential of the dipeptide 5a-e and 6a-e.

\begin{tabular}{|c|c|c|c|c|c|}
\hline No. & $\begin{array}{c}\mathrm{P}_{2} \\
\mathrm{P}_{2} \text { = tert-butylamino }\end{array}$ & $\begin{array}{c}\% \text { HIV } \\
\text { protease }^{\text {inhibition }}{ }^{\text {a }} \\
5 \mu \mathrm{M}\end{array}$ & No. & $\begin{array}{c}\mathrm{P}_{2} \\
\mathrm{P}_{2} `=2-\text { methylbenzylamino }\end{array}$ & $\begin{array}{c}\% \text { HIV } \\
\text { protease } \\
\text { inhibition }^{\text {a }} \\
5 \mu \mathrm{M} \\
\end{array}$ \\
\hline $5 \mathbf{a}$ & & 21.1 & $6 a$ & & 53.2 \\
\hline $5 b$ & & 89.9 & $\mathbf{6 b}$ & & 98.3 \\
\hline $5 c$ & & 25.7 & 6c & & 69.6 \\
\hline $5 d$ & & 12.6 & 6d & & 59.6 \\
\hline $5 e$ & & 8.0 & $6 \mathbf{e}$ & & 64.8 \\
\hline
\end{tabular}

KNI-577 : 87.6\% (50 nM), KNI-727 : 95.9\% (50 nM). 
when 2-methylbenzyl replaced tert-butyl group. This was illustrated by results on Table 4 , since the activity of $\mathbf{5 c - e}$ is much less than their analogs 6c-e. It seems that $\mathrm{S}_{2}{ }^{\prime}$ site, where $\mathrm{P}_{2}$ ' moiety interacts can tolerate less bulky and more polar group than tert-butyl moiety.

In general the dipeptides obtained in our study were found less active than the leads KNI-577 and KNI-727.

\section{Conclusion}

The introduction of 2-methylbenzylmoiety as $\mathrm{P}_{2}$ `ligand made a considerable improvement of the HIV inhibitory activity at level $5 \mu \mathrm{M}$ in comparison to the corresponding tert-butyl containg analogs.

Generally the synthesized compounds showed moderate HIV protease inhibitory activity matched with the lead compounds KNI-577 and KNI-727.

\section{REFERENCES}

1- E. De Clercq, J. Med. Chem., 38, 24912517 (1995).

2- Y. Kiso, Biopolymers (Peptide Science), 40, 235-244 (1996).

3- S. Yamaguchi, T. Mitoguchi, S. Nakata, T. Kimura, K. Akaji, S. Nojima, H. Takaku, T. Mimoto and Y. Kiso, Peptide Chemistry 1996, 297-300; C. Kitada (Ed.) Protein research Foundation, Osaka (1997).
4- Y. Kiso, S. Yamaguchi, H. Matsumoto, T. Mimoto, R. Kato, S. Nojima, H. Takaka, T. Fukazawa, T. Kimura, K.Akaji, (Proceedings of the $15^{\text {th }}$ American Peptide Symposium, Nashville, Abst. No. L023, 14-19 June (1997).

5- H. M. M. Abdel-Rahman, Ph. D. Thesis, Assiut University, Egypt (1999).

6- M. M. Sheha, N. M. Mahfouz, H. Y. Hassan, A. F. Youssef, T. Mimoto and Y. Kiso, Eur J. Med. Chem., 35, 887-894 (2000).

7- R. Nishizawa, T. Saino, T. Takita, H. Suda, T. Aoyagi, and H. Umezawa, J. Med. Chem., 20, 510-515 (1977).

8- M. Schutkowski, C. Mrestani-Klaus and K. Neubert, Int. J. Peptide Protein Res., 45,257-265 (1995).

9- T. Mimoto, R. Kato, H. Takaku, S. Nojima, K. Terashima, S. Misawa, T. Fukazawa, T. Ueno, H. Sato, M. Shintani, Y. Kiso and H. Hayashi, J. Med. Chem., 42, 1789-1802 (1999).

10- D. Hudson, J. Org. Chem., 53, 617-624 (1988).

11- T. Miyazawa, T. Yamada and S. Kuwata, Bull. Chem. Soc. Jpn., 61, 606-608 (1988).

12- A. S. Kende, D. Scholz and J. Schneider, Synth. Commun., 8, 59-63 (1978).

13- J. Dudash, Jr., J. Jiang, S. C. Mayer and M. M. Joullie, Synth. Commun, 23, 349356 (1993). 